OMAE2014-24441

\title{
EVANESCENT WAVE REDUCTION USING A SEGMENTED WAVEMAKER IN A TWO DIMENSIONAL WAVE TANK
}

\author{
lain Keaney * \\ Centre for Ocean Energy Research \\ NUI Maynooth \\ Maynooth, Co. Kildare, Ireland \\ iain.keaney@nuim.ie
}

\author{
Ronan Costello \\ Centre for Ocean Energy Research \\ NUI Maynooth \\ Maynooth, Co. Kildare, Ireland \\ ronan.costello@eeng.nuim.ie
}

\author{
John V. Ringwood \\ Centre for Ocean Energy Research \\ NUI Maynooth \\ Maynooth, Co. Kildare, Ireland \\ john.ringwood@eeng.nuim.ie
}

\begin{abstract}
The concept of a segmented wavemaker, in a two dimensional tank, has been investigated analytically to see if it can reduce the effect of parasitic evanescent waves in a wave tank. Evanescent waves can contaminate test areas in tanks leading to unreliable results, but are typically avoided by establishing the test area two to three times the water depth away from the wavemaker. This space requirement can be quite restrictive in terms of the necessary tank size and, with the increasing interest in off-shore renewable energy, many technology developers may not be able to afford a workspace large enough to accommodate a long tank. Previously, flexible wavemakers have been designed to tackle the problem and, in some cases have proven very effective in eliminating evanescent waves at the wavemaker's "tuned" frequency. However, flexible wavemakers have been shown to have little benefit in modeling panchromatic seas. Discussed here is the linear potential theory of a segmented wavemaker designed to reduce the evanescent waves in a tank over a large frequency range. Each segment in the segmented wavemaker is programmed with an individual stroke, allowing the system to best approximate the horizontal displacement of the fluid over the depth of the water in a naturally occurring wave. A comparison of the influence of evanescent waves created by segmented wavemakers, piston and hinged paddle wavemakers, on the free surface elevation is presented.
\end{abstract}

\footnotetext{
*Address all correspondence to this author.
}

\section{NOMENCLATURE}

$\phi \quad$ Velocity potential of fluid

$\varphi \quad$ Complex coefficient of of proportionality

$u_{j} \quad$ Velocity of a body in the $j^{t h}$ mode of motion

$c_{n} \quad$ Coefficients of the $n^{\text {th }}$ term of the velocity potential

$Z_{n}(z) \quad$ Functions describing the variation of the fluid's motion over depth for the $n^{\text {th }}$ term of the velocity potential

$k \quad$ Wavenumber for the progressive wave

$m_{n} \quad$ Wavenumber for the $n^{\text {th }}$ evanescent mode

$x \quad$ Horizontal cartesian coordinate

$z \quad$ Vertical cartesian coordinate

$N_{n} \quad$ Normalisation function for the $n^{\text {th }}$ solution to the dispersion relation

$h \quad$ Still water depth

$c(z) \quad$ Normalised profile of the segment's displacement over depth

$v \quad$ Velocity of the fluid

$\omega \quad$ Angular frequency

$g \quad$ Acceleration due to gravity

$\eta \quad$ Free surface elevation

$Z_{i, j} \quad$ Component $(i, j)$ of the radiation impedance matrix

$\rho \quad$ Density of the fluid

v Generalised velocity vector

$R(\omega) \quad$ Radiation damping

$m(\omega) \quad$ Added mass

$F_{c} \quad$ Constrained radiation force

$u^{q} \quad$ Velocity of the $q^{\text {th }}$ segment

$s \quad$ System's independent speed 


$\begin{array}{ll}\mathbf{F} & \text { Radiation force vector } \\ N & \text { Number of segments in a system } \\ \mathbf{P} & \text { Velocity transformation vector } \\ \mathbf{Z} & \text { Radiation impedance matrix } \\ \mathbf{u} & \text { Wavemaker's velocity vector } \\ Z_{c} & \text { Constrained radiation impedance } \\ R_{c}(\omega) & \text { Constrained radiation damping } \\ m_{c}(\omega) & \text { Constrained added mass } \\ S_{q} & \text { Stroke of the } q^{\text {th }} \text { segment }\end{array}$

\section{INTRODUCTION}

The growing interest in off-shore energy has seen many new research groups emerging in both academia and industry in recent years. However, a number of these groups do not have in-house tank testing capabilities and instead incur huge costs when performing tank testing in off-site facilities, whereas, the cost of housing a wave tank can be considerably less over time and allows the group the freedom to preform frequent tank tests. Consequently, many of these groups have found that progress is suffering as a result. One difficulty preventing groups from having an in-house wave tank is the lack of space necessary. In a wave tank a considerable amount of space is not used for testing devices but rather for allowing the wave field to resolve to a natural form. This paper aims to reduce the size of a wave tank by investigating the methods used for generating waves.

A wavemaker forces a motion on the fluid which is different from the fluid motion in a naturally occurring wave, leading to a distortion in the wave field near the wavemaker. This distortion can be explained as an infinite number of evanescent waves, which appear on the free surface as standing waves, superimposed on the radiating wave. These evanescent waves are so called as they vanish with distance away from the wavemaker. The typical rule to avoid this distortion when performing tank testing is to leave a distance of two to three times the water depth between the wavemaker and the test area (Dean and Dalrymple [1]). Furthermore, if the waves are being canceled out by an active absorbing wavemaker at the far end of the tank, the same provisions of leaving a distance of two to three times the water depth between the test area and the wave absorber should be adhered to. Since the motion of the fluid in a naturally occurring wave decreases exponentially over depth, the perfect wavemaker would be able to create a similar disturbance in the fluid. However, this exponential is dependant on the wavenumber and thus, the ideal wavemaker must be able to change its geometry for each frequency. Banner and Peirson [2] used such a wavemaker, based on a cantilever design, and Biesel and Suquet [3] reported on a similar design using a flexible membrane actuated at several points over the depth by geared wheels. In both cases, however, the wavemakers were geometrically "tuned" to a particular frequency. A CFD investigation into such a wavemaker (Maguire [4]) showed that at either side of the system's tuned frequency the distortion increases quickly and that, over a large range of frequencies, the design shows little to no benefit over the simpler piston or hinged paddle designs.

Typically, to reduce the effect of the distortion, wave tanks are designed to suit a particular type of testing; for instance, a piston wavemaker is used for modeling shallow water waves, where the relative depth value, given as the wavenumber, $k$, times the average water depth is $k h<\pi$. Similarly, a hinged paddle is used to model deep water waves, where $k h>\pi$, (Dean and Dalrymple [1]). The concept of the segmented wavemaker, as illustrated in Figure 1, was proposed by Naito [5] as a method which can approximate the fluid motion in any naturally occurring wave. Presented in this paper is an investigation into the distortion of the free surface from segmented systems, ranging from a single segment system to a ten segment system. The segments in a system are all of equal lengths and operate only in surge mode; hence, the single segment system is a typical piston wavemaker. The hydrodynamic coefficients of each system are also presented and the results of the segmented wavemaker are compared to those of a single hinged paddle.

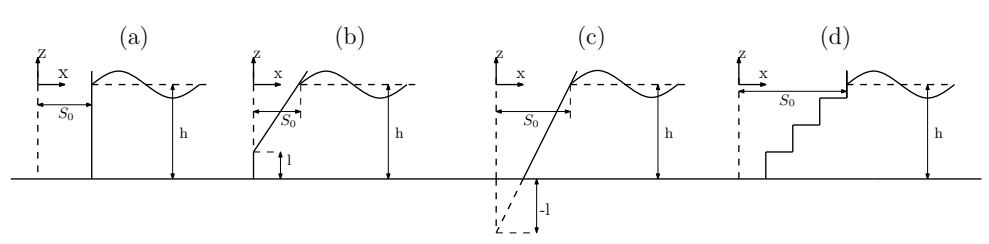

FIGURE 1. (a): Piston wavemaker, $(b)$ : hinged paddle wavemaker where the hinge is fixed above the tank floor, $(c)$ : hinged paddle wavemaker with a virtual hinge located below the tank floor, $(d)$ : segmented wavemaker.

\section{Wavemaker theory}

\subsection{Linear potential theory}

Wavemaker theory, first developed by Havelock [6], has been extensively documented in the literature; here we will follow the methodology used by Falnes [7]. This study considers a two dimensional wave tank with a depth, $h$, of $0.6 \mathrm{~m}$. The cartesian coordinate system is used where the $x$-axis is horizontal, while the wavemaker's mean position is at $x=0$ and the $z$ axis points vertically upwards with the mean free surface level at $z=0$, as shown in Figure 1, where $S_{0}$ denotes the wavemaker's stroke.

Using potential theory, the fluid's velocity can be expressed as the gradient of a scalar potential function called the velocity 
potential,

$$
\vec{v}=\nabla \phi
$$

The velocity potential, $\phi$, is given as:

$$
\hat{\phi}=\sum_{j=1}^{6} \varphi_{j} \hat{u}_{j}
$$

where $u_{j}$ is the wavemaker's velocity in the $j^{\text {th }}$ degree of freedom, and the hat, $\hat{,}$ denotes the complex amplitude of a function. The complex coefficient of proportionality, $\varphi$, which is of the form:

$$
\varphi=X(x) Z(z)
$$

must satisfy the governing equation and boundary conditions on the fluid domain. As potential theory assumes that the fluid is incompressible, then the law of conservation of mass gives the governing equation as:

$$
\nabla \vec{v}=0
$$

which is the Laplace equation. The relavent boundary conditions on the fluid are:

- The bottom boundary condition on the tank floor:

$$
\left.\frac{\partial \varphi}{\partial z}\right|_{z=-h}=0 .
$$

- The free surface boundary condition:

$$
\omega^{2} \varphi+g \frac{\partial \varphi}{\partial z} \mid=0
$$

where $\omega$ is the angular frequency and $g$ is the acceleration due to gravity. The free surface boundary condition has been linearly approximated about the position $z=0$.

- The boundary condition on the surface of the wavemaker:

$$
\left.\frac{\partial \varphi_{j}}{\partial x}\right|_{x=0}=c_{j}(z)
$$

which has been linearly approximated about the position $x=0$. The function $c_{j}(z)$ is the profile of the wavemaker's normalised displacement, in the $j^{\text {th }}$ degree of freedom, over the $z$-axis. In the case of the segmented wavemaker presented here, each of the segments is restricted to operating only in surge, where $j=1$, so from here on the subscript will be dropped and the profile for each segment set as: $c(z)=1$.

The general solutions to the Laplace equation, Equation (4), are:

$$
X(x)=c_{+} e^{k x}+c_{-} e^{-k x},
$$

and

$$
Z(z)=c_{+} e^{k z}+c_{-} e^{-k z},
$$

where $k$ is the wavenumber. Considering that we are only interested in the wave propagating in the positive $x$ direction away from the wavemaker, i.e., no reflected waves, the second term in Equation (8) can be ignored, giving $X(x)=c e^{k x}$. By applying the bottom boundary condition, Equation (5), to Equation (9) and after carrying out some algebra (Falnes [7]) the function $Z(z)$ can be found as:

$$
Z(z)=N^{-\frac{1}{2}} \cosh [k(h+z)]
$$

where the normalisation constant $N$ is:

$$
N=\frac{1}{2}\left[1+\frac{\sinh (2 k h)}{2 k h}\right]
$$

By applying the free surface boundary condition, Equation (6), to $Z(z)$ the dispersion relation is derived:

$$
\omega^{2}=g k \tanh (k h)
$$

for which there is only one real solution, $k$. However, there is an infinite number of imaginary solutions that satisfy the dispersion relation, and these are the wavenumbers of the evanescent waves. So the dispersion relation, Equation (12), can be more generally writen as:

$$
\omega^{2}=-g m_{n} \tan \left(m_{n} h\right)
$$

where the first solution is $m_{0}=i k$. Similarly the general forms 
of Equations (10) and (11) are:

$$
Z_{n}(z)=N_{n}^{-\frac{1}{2}} \cos \left[m_{n}(h+z)\right]
$$

and

$$
N_{n}=\frac{1}{2}\left[1+\frac{\sin \left(2 m_{n} h\right)}{2 m_{n} h}\right] .
$$

Since all possible solutions must exist then $\varphi$ can be expressed as the superposition:

$$
\varphi_{1}=\sum_{n=0}^{\infty} c_{n} Z_{n}(z) e^{m_{n} x}
$$

where the first term in the summation coorespondes to the progressive wave and all the other terms represent evanescent waves. The coefficient $c_{n}$ can be found by applying the wavemaker boundary condition, Equation (7), to $\varphi$ :

$$
c(z)=\left.\frac{\partial \varphi}{\partial x}\right|_{x=0}=\sum_{n=0}^{\infty} m_{n} c_{n} Z_{n}(z)
$$

multiplying across by $Z_{m}^{*}(z)$, integrating over the $z$-axis and using the orthogonal condition (Falnes [7]), the $c_{n}$ coefficient can be found as:

$$
c_{n}=\frac{1}{m_{n} h} \int_{-h}^{0} c(z) Z_{n}^{*}(z) d z .
$$

The complex conjugate of a function is denoted by ${ }^{*}$. The complex amplitude of the free surface is given by,

$$
\hat{\eta}=-\frac{i \omega}{g}[\hat{\phi}]_{z=0},
$$

The distortion of the free surface is obtained as the additional amplitude of the free surface due to the evanescent waves, $\hat{\eta}_{\text {evan }}$, as a percentage of the free surface elevation due to the radiating wave, $\hat{\eta}_{0}$, where,

$$
\hat{\eta}_{\text {evan }}=-\frac{i \omega}{g}\left[\hat{u}_{1} \sum_{n=1}^{\infty} c_{n} Z_{n}(z)\right]_{z=0}
$$

and

$$
\hat{\eta}_{0}=-\frac{i \omega}{g}\left[\hat{u}_{1} c_{0} Z_{0}(z)\right]_{z=0}
$$

The distortion is then calculated from:

$$
\text { Distortion }=\frac{\left|\hat{\eta}_{\text {evan }}\right|}{\left|\hat{\eta}_{0}\right|} \times \frac{100}{1}
$$

\subsection{Radiation impedance}

The component of the radiation impedance matrix due to the wave being radiated by the wavemaker's $j^{t h}$ segment acting on the $i^{\text {th }}$ segment is given as:

$$
Z_{i j}(\omega)=-i \omega \rho \int_{b}^{a} \varphi_{j} n_{i} d z
$$

where the segments surface is defined over the region $-a>z>-b$, and $n_{i}$ is the unit vector pointing normally into the fluid domain from the surface of the $i^{t h}$ segment. The radiation damping and added mass of the wavemaker can be found from the real and imaginary parts of the radiation impedance respectively as,

$$
R(\omega)=\operatorname{Re}\{Z(\omega)\}
$$

and

$$
m(\omega)=\frac{1}{\omega} \operatorname{Im}\{Z(\omega)\}
$$

\subsection{Equivalent constrained parameters}

In order to be able to compare the hydrodynamic coefficients of systems with different numbers of segments, the NewtonEuler equations of motion with Eliminated Constraints (NE-EC) are used, to reduce the number of degrees of freedom in the multi-body system. This provides frequency dependant scalar values of the hydrodynamic coefficients for each system, refered to here as equivelent constrained parameters, which are then comparable to the hydrodynamic coefficients of a single body system. The NE-EC is given (O'Cathain [8]) as:

$$
F_{c}=\sum_{q=1}^{N} \frac{\partial u_{q}}{\partial s} \mathbf{F}=\mathbf{P F} .
$$


Here, $F_{c}$ is the $N$-segment system's equivelent constrained force, while $u_{q}$ is the velocity of the $q^{t h}$ segment. The segmented wavemaker's independent velocity, $s$, is set to the velocity of the segment closest to the free surface, $s=u_{1}$. The partial derivative, $\frac{\partial u_{q}}{\partial s}$, is the velocity of the $q^{t h}$ segment with respects to the independent velocity. The force vector, $\mathbf{F}$, is a column vector where the $q^{\text {th }}$ component is the radiation force on the $q^{t h}$ segment and the velocity transform vector, $\mathbf{P}$, is:

$$
\mathbf{P}=\left(\frac{\partial u_{1}}{\partial s}, \ldots, \frac{\partial u_{q}}{\partial s}, \ldots, \frac{\partial u_{N}}{\partial s}\right)
$$

The segmented wavemaker's generalised velocity vector is given as:

$$
\mathbf{v}=\mathbf{P}^{T} s
$$

The radiation force on the wavemaker system is obtained by the product of the radiation impedance and the wavemaker's velocity; in matrix form this is:

$$
\mathbf{F}=\mathbf{Z v}
$$

Similarly, this can be expressed in the form of the equivalent constrained parameters:

$$
F_{c}=Z_{c} s
$$

Substituting Equations (29) and (28) into Equation (26) and equating with Equation (30), the equivalent constrained radiation impedance, $Z_{c}$, can be obtained as:

$$
Z_{c}=\mathbf{P Z} \mathbf{P}^{T}
$$

Thus, the equivalent constrained hydrodynamic coefficients are found to be:

$$
R_{c}=\mathbf{P R} \mathbf{P}^{T},
$$

and

$$
m_{c}=\mathbf{P} \mathbf{m} \mathbf{P}^{T}
$$

where $\mathbf{R}$ and $\mathbf{m}$ are the radiation damping and added mass matrices, respectively.

In order to minimise the effect of the evanescent waves a control system should be implemented, which uses feedback to calculate the optimal velocities of the segmentes relative to each other, i.e., the velocity transform vector $\mathbf{P}$, to absorb any reflected waves. However, as this paper makes the simplification of assuming that there are no reflected waves; the use of a control system can be avoided by prescribing the $q^{\text {th }}$ segment with a stroke that matches the displacement of the fluid in a progressive wave, giving the stroke:

$$
S_{q}=\frac{\cosh \left[k\left(h+\frac{h(q-1)}{N}\right)\right]}{\cosh (k h)} .
$$

In the case of the segment closest to the free surface, where $q=1$, the stroke is: $S_{q}=1$. Thus, the velocity of the $q^{\text {th }}$ segment is:

$$
u_{q}=i \omega \frac{\cosh \left[k\left(h+\frac{h(q-1)}{N}\right)\right]}{\cosh (k h)}
$$

and the velocity transformation vector, $\mathbf{P}$, can be found to be:

$$
\mathbf{P}=\left(1, \ldots, \frac{\cosh \left[k\left(h+\frac{(q-1) h}{N}\right)\right]}{\cosh (k h)}, \ldots, \frac{\cosh \left[k\left(h+\frac{(N-1) h}{N}\right)\right]}{\cosh (k h)}\right)
$$

\section{Results}

\subsection{Wave field distortion}

The distortion of the wave field, given in Equation (22), is plotted as a function of the relative depth, $k h$, for ten different segmented systems as well as the hinged paddle in Figure 2 where it is evident that, by increasing the number of segments in the system, the wave field's distortion can be significantly reduced. From Figure 2, it is clear that the more segments there are in a system, the less benefit is achieved by including an additional segment. However, it must be appreciated that the three segment system offers very little benefit over the simpler design of the hinged paddle system for $k h>3$. However, for $k h<1.3$, the distortion caused by the single segment system is less than $10 \%$, agreeing with the common practice of using a piston system for modeling shallow water waves and a hinged paddle for modeling deep water waves.

The normalised distance from the wavemaker of $10 \%, 5 \%$ and $1 \%$ distortion for all the wavemaker systems are shown in Figures 3, 4 and 5, respectively. What is clear from Figures 3, 4 5 , and 2 is that the hinged paddle, and some of the segmented 


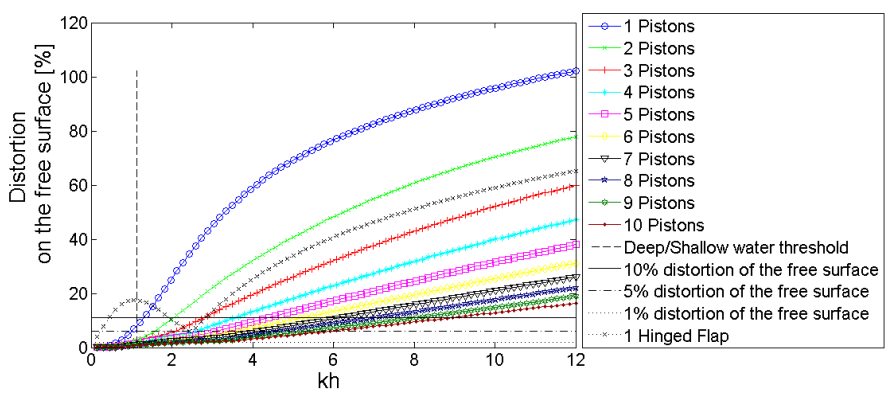

FIGURE 2. The distortion of the free surface at $x=0$ as a function of relative depth, $k h$.

wavemaker systems have an optimal, or "tuned", frequency. This is due to some of the evanescent waves undergoing a phase shift of $\pi$ radians. This phase shift is caused by a change of sign in the $c_{n}$ coefficient, resulting in destructive interference. By implementing a control system, it may be possible to induce this phase shift for a range of frequencies by controlling the stroke of each segment. Effectively, this could use some of the evanescent waves to cancel out the other evanescent waves, thus, reducing the distortion for a larger range of frequencies.

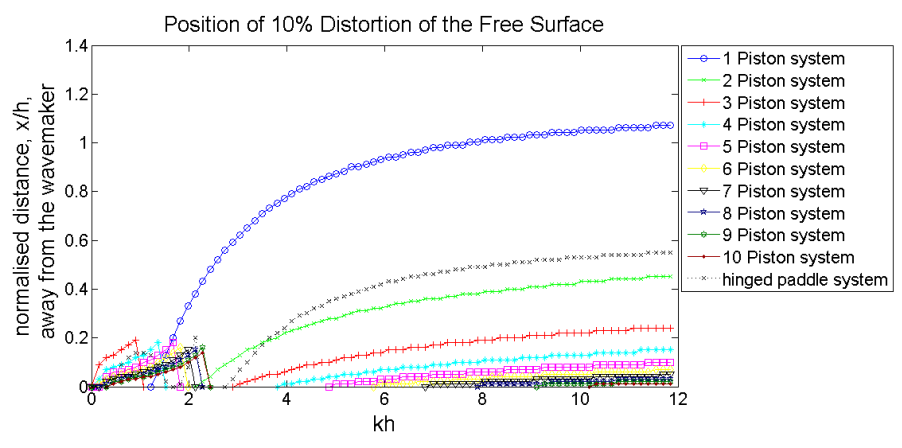

FIGURE 3. The normalised distance, $x / h$, from the wavemaker, where $10 \%$ distortion occurs over the range of relative depth, $k h$, values.

\subsection{Hydrodynamic coefficients}

The equivalent constrained radiation damping, given by Equation (32), is plotted as a function of the relative depth in Figure 6 for all the wavemaker systems. It can been seen that, the more segments there are in the system, the harder it is for the wavemaker to radiate large amplitude waves. This is due to the wavemaker displacing less fluid and is accounted for when cal-

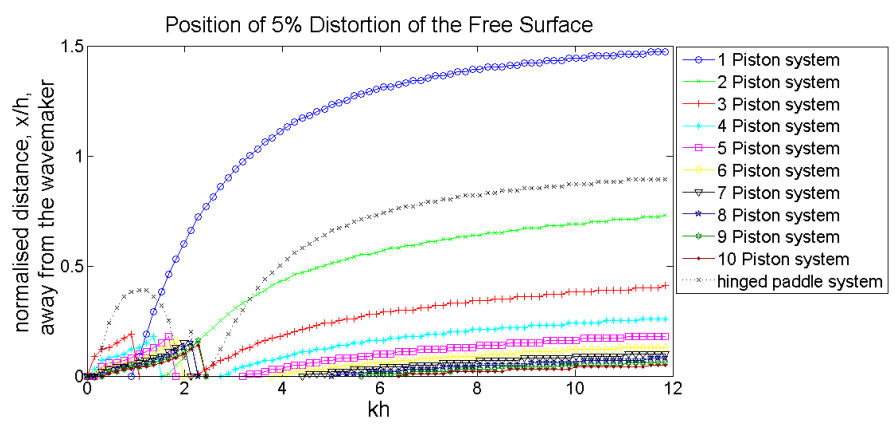

FIGURE 4. The normalised distance, $x / h$, from the wavemaker, where $5 \%$ distortion occurs over the range of relative depth, $k h$, values.

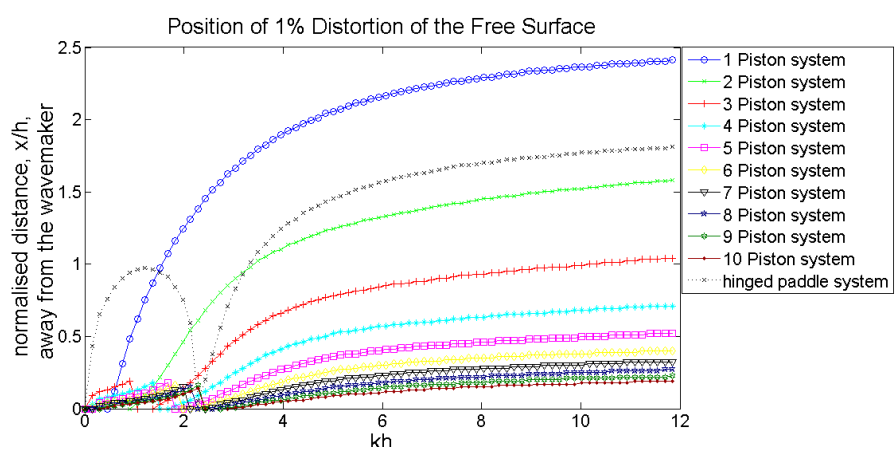

FIGURE 5. The normalised distance, $x / h$, from the wavemaker, where $1 \%$ distortion occurs over the range of relative depth, $k h$, values.

culating the distortion in Figures 2 to 5 by adjusting the stroke of each system so that the amplitude of the radiated wave equals that generated by a single segment system with a unit stroke.

As expected, the equivalent constrained added mass of the wavemaker system, Equation (33), which is related to the distortion of the wave field, decreases as more wavemaker segments are included; this is illustrated in Figure 7.

\subsection{Hydrodynamic model verification}

In order to show that the hydrodynamics were calculated accurately according to linear potential theory, the horizontal velocity components, due to the first 150 terms of Equation (16), of the fluid on the surface of the wavemaker, for a wave of: $k=12 \mathrm{~m}^{-1}$, have been plotted against the $z$-axis in Figure 8 for the single, two, and ten segment wavemaker systems. It can be seen in Figure 8 that the fluid's horizontal velocity is close to that of the wavemaker. In fact, if the infinite number of evanescent waves were to be included when calculating the velocity potential in Equation (16), the fluid's horizontal velocity at $x=0$, and that of the wavemaker, would be equal. All the results presented 


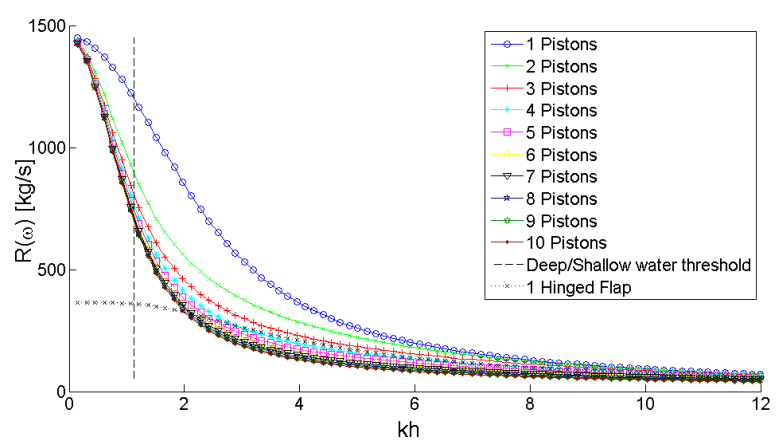

FIGURE 6. Constrained radiation damping as a function of relative depth, $k h$.

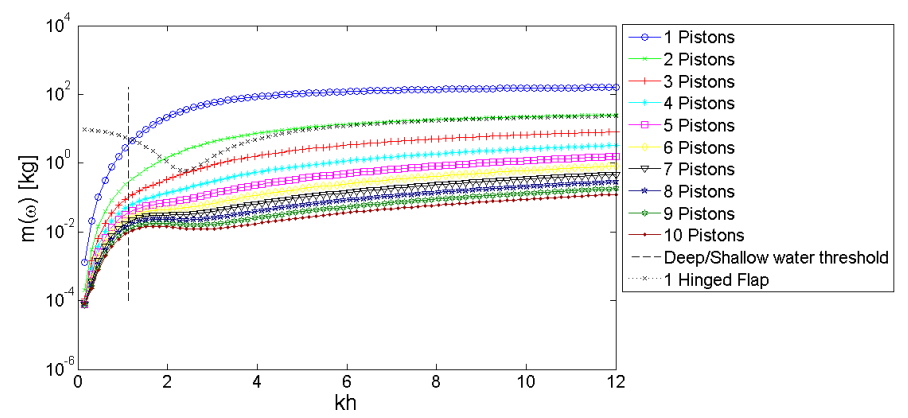

FIGURE 7. Constrained added mass as a function of relative depth, $k h$.

in this paper, except for Figure 8, have been calculated from a truncated velocity potential limited to the first fifty terms of the summation in Equation (16). The ratio of the free surface elevation amplitude at the wavemaker, including the first ten, fifty and one hundred terms, to the free surface elevation amplitude caused by the radiated wave, has been plotted as a function of frequency for both the single and ten segment systems in Figure 9. It is evident from Figure 9 that just including the first ten terms of the summation in Equation (16) is not enough to provide an accurate calculation of the velocity potential in the case of the single segment system; however, it is also clear that there is little point in including more than the first fifty terms.

\section{Conclusion}

The quality of the wave field created by a segmented wavemaker consisting of one to ten segments was investigated. The level of distortion was assessed by the amplitude of the evanescent waves as a precentage of the amplitude of the radiating wave. The results presented here show clearly that, by increasing
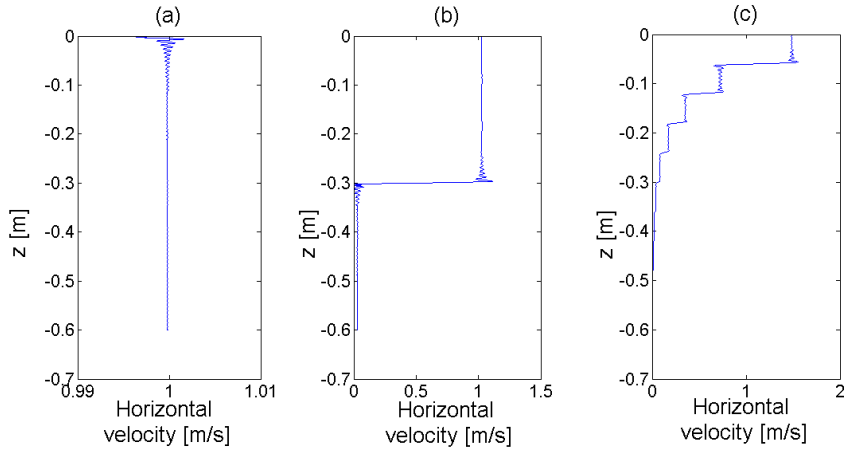

FIGURE 8. The horizontal velocity components for: (a) single, (b) two and (c) ten segment system, plotted over depth.

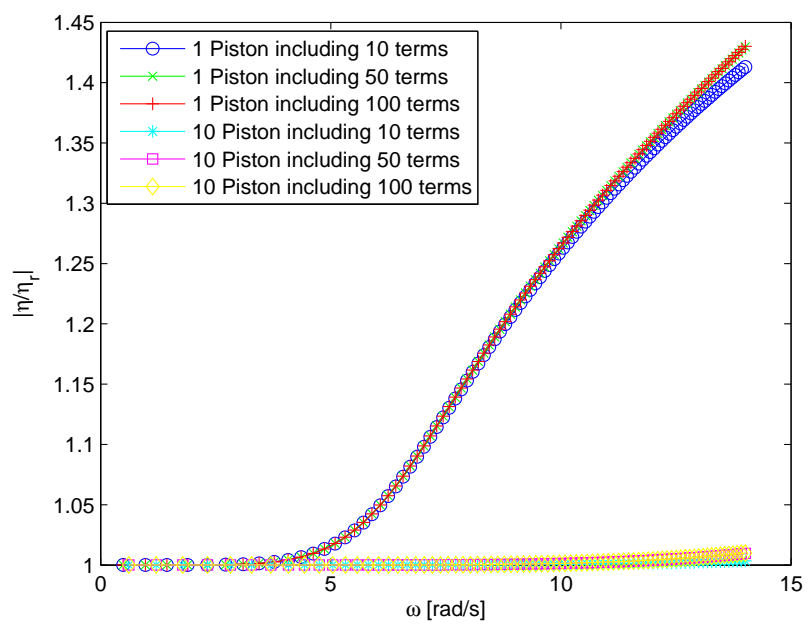

FIGURE 9. The absolute value of the ratio of the free surface elevation including the first ten, fifty and one hundred terms of the velocity potential to the free surface elevation due to the radiated wave at $x=0$ as a function of frequency, for both the single and ten segment system.

the number of segments in the wavemaker, the distortion of the wave field can be greatly reduced. However, the benefit of an additional segment decreases as more segments are added. The results presented show that, for deep water waves, the distortion to the wave field created by the simple hinged paddle wavemaker is between that created by the more complicated two and three segment wavemakers. However, it is worth noting that the segments are restricted to operating in surge. Following on from this, further improvements could be made to the segmented system, such as allowing each segment to operate in pitch as well as surge, and optimising the lengths of the individual segments, both of which could make the segmented wavemaker much more effective. 


\section{Acknowledgements}

The authors are grateful for the financial support provided by Enterprise Ireland and Omey Labs Ltd., under the Innovation Voucher funding scheme.

\section{REFERENCES}

[1] Dean, R. G., and Dalrymple, R. A., 1984. Water Wave Mechanics For Engineers and Scientists, 1st ed. World Scientific, Singapore.

[2] Banner, M. L., and Peirson, W. L., 2007. "Wave breaking onset and strength for two-dimensional deep-water wave groups". Journal of Fluid Mechanics, 585, pp. 93-115.

[3] Biesel, F., and Suquet, F., 1951. 'Laboratory wavegenerating Apparatus". St. Anthony Falls Hydraulic Laboratory, University of Minnesota, Minneapolis.

[4] Maguire, A. E., 2011. "Geometric design considerations and control methodologies for absorbing wavemakers". PhD thesis, University of Edinburgh.

[5] Naito, S., 2006. "Wave Generation and Absorption -Theory and Application". In International Offshore and Polar Engineering Conference, Vol. 4, San Fancisco, USA.

[6] Havelock, T. H., 1929. "Forced surface waves on water". Philosophical Magazine Series 7, 8(51), pp. 569-576.

[7] Falnes, J., 2002. Ocean Waves and Oscillating Systems. Cambridge University Press, Cambridge.

[8] ÓCatháin, M., Leira, B. J., Ringwood, J. V., and Gilloteaux, J.-C., 2008. "A modelling methodology for multi-body systems with application to wave-energy devices". Ocean Engineering, 35(13), Sept., pp. 1381-1387. 